\title{
Tamaño óptimo de muestra para estimar la distribución de tallas y proporción de juveniles de anchoveta por lance a bordo de la flota de cerco peruana
}

\section{Optimum sample length for estimating anchovy size distribution and the proportion of juveniles per fishing set for the Peruvian purse-seine fleet}

\section{Rocío Joo * y Erich Díaz}

Instituto del Mar del Perú (IMARPE) esquina Gamarra y General Valle S/N Chucuito, Callao, Perú.

*Autor para correspondencia.

E-mail Rocío Joo: rocio.joo@gmail.com

E-mail Erich Díaz: ediaz@imarpe.gob.pe

\begin{abstract}
Resumen
La distribución de tallas de las capturas es una fuente de información esencial para la estimación del crecimiento y la dinámica espacio-temporal de las cohortes. La distribución de tallas de las capturas se estima a partir de muestras de individuos capturados en los lances. Este trabajo estudia la cantidad óptima de individuos a muestrear en cada lance para obtener una muestra representativa de las tallas del lance, y de la proporción de juveniles en el lance. Para ello, se utilizan datos de tallas de diferentes lances en la pesca de anchoveta peruana (Engraulis ringens) registrados por observadores a bordo del Programa Bitácoras de Pesca del Insti-tuto del Mar del Perú, y remuestreos a partir de esos datos. Finalmente proponemos un tamaño de muestra óptimo que permite obtener estimaciones robustas de tallas y proporción de juveniles. Aunque este trabajo se aplicó a la pesca de anchoveta, el procedimiento es aplicable a cualquier pesquería, para muestreos bio-métricos a bordo o en tierra.
\end{abstract}

Palabras claves: distribución de tallas; proporción de juveniles; tamaño de muestra; observadores a bordo;pesquería de anchoveta; Engraulis ringens.

\section{Abstract}

The length distribution of catches represents a fundamental source of information for estimating growth and spatiotemporal dynamics of cohorts. The length distribution of caught is estimated based on samples of catched individuals. This work studies the optimum sample size of individuals at each fishing set in order to obtain a representative sample of the length and the proportion of juveniles in the fishing set. For that matter, we use anchovy (Engraulis ringens) length data from different fishing sets recorded by observers at-sea from the On-board Observers Program from the Peruvian Marine Research Institute. Finally, we propose an optimum sample size for obtaining robust size and juvenile estimations. Though the application of this work corresponds to the anchovy fishery, the procedure can be applied to any fishery, either for on board or inland biometric measurements.

Keywords: length distribution; proportion of juveniles; sample size; on-board observers; anchovy fishery; Engraulis ringens.

Citación:

Joo R. y E. Díaz. 2017. Tamaño óptimo de muestra para estimar la distribución de tallas y proporción de juveniles de anchoveta por lance a bordo de la flota de cerco peruana.. Revista peruana de biología 24(1): 059 - 066 (Abril 2017). doi: http://dx.doi.org/10.15381/rpb.v24i1.13112
Información sobre los autores:

RJ: realizó la escritura del manuscrito, el diseño de la metodología empleada, los algoritmos y cálculos para el trabajo. ED: generó la pregunta de investigación del trabajo, coordinó la toma de información, participó activamente en las discusiones y en la redacción del trabajo.

Los autores no incurren en conflictos de intereses.

Fuentes de financiamiento:

Proyecto PpR "Observación y evaluación en tiempo real del subsistema pelágico del Ecosistema de la Corriente de Humboldt utilizando como plataforma la flota de cerco" del IMARPE, financiado con recursos de Derechos de Pesca (RM-N ${ }^{\circ}$ 235-2013-PRODUCE).

Proyecto $\mathrm{PpR}$ "Estimación de parámetros biológico-pesqueros para el manejo sostenible de los recursos marinos"del IMARPE, financiado con recursos de Derechos de Pesca (RM-350-2013-PRODUCE). $\begin{array}{ll}\text { Presentado: } & 31 / 12 / 2015 \\ \text { Aceptado: } & 25 / 09 / 2016 \\ \text { Publicado online: } & \text { 20/04/2017 }\end{array}$ 


\section{Introducción}

La distribución de tallas de las capturas es una de las fuentes de información más importantes para inferir el estado más reciente de un recurso explotado. A partir de las tallas capturadas se puede estimar el crecimiento y su dinámica espacio-temporal, los patrones de selectividad, el tamaño del stock y la magnitud de la mortalidad por pesca y total a la que está siendo sometida (Pauly \& Morgan 1987, Quinn \& Derisso 1999). Además, a partir de la distribución por tallas de las capturas se puede estimar la proporción de juveniles que es capturada por las flotas pesqueras y tomar medidas para evitar caer en la sobreexplotación sobre el crecimiento (Di Natale et al. 2002, Suuronen \& Sardá 2007).

El Programa Bitácoras de Pesca (PBP) de observadores a bordo de la flota de cerco del Instituto del Mar del Perú (IMARPE) recolecta, entre otra cosas, datos de tallas de los principales recursos pelágicos capturados en el mar peruano (i.e. anchoveta Engraulis ringens, jurel Trachurus murphyi, caballa Scomber japonicus). A partir de esos datos se estima la frecuencia por tallas de las capturas y los porcentajes de juveniles de los recursos pelágicos capturados por la flota de cerco. La frecuencia por tallas es generalmente utilizada a una escala mensual o anual para estimar el crecimiento de los peces pelágicos (Palomares et al. 1987, Díaz 2013), y para la evaluación de los stocks a partir de los análisis de población virtual (Pauly et al. 1987) o modelos estocásticos de dinámica poblacional (Oliveros-Ramos \& Díaz 2015). El porcentaje de juveniles es utilizado a una escala diaria para monitorear y vedar las áreas con alta incidencia de juveniles (e.g. RM N² 215-2015-PRODUCE).

Para la estimación de ambas variables, se realizan muestreos biométricos, procedimiento que consiste en la selección de un conjunto de individuos de una determinada especie y en el registro de las tallas de los individuos seleccionados. Este muestreo se hace por lance de pesca y la muestra se recoge en el momento del lance que el observador encuentra más oportuno (Bouchon et al. 1998). En la pesca de anchoveta (que representa el 95\% de las capturas de la flota pelágica; PRODUCE 2014), los manuales de recolección de datos (Bouchon et al. 1998, 2001) indican que para el muestreo biométrico se debe recolectar un balde de $2 \mathrm{~kg}$ de anchoveta y seleccionar de manera aleatoria entre 120 a 160 individuos. Luego, se debe utilizar un ictiómetro para medir la longitud total de cada pez, lo cual permite obtener tallas entre los 3.0 y $20.0 \mathrm{~cm}$ con intervalos de clase con amplitud de 0.5 $\mathrm{cm}$. En la práctica, los observadores suelen tomar muestras más grandes que las mínimas requeridas: para los ańos 2010 - 2015, la mediana del número de individuos muestreados fue 178. Un tamaño de muestra más grande debería aumentar la precisión de las estimaciones realizadas respecto a las tallas. Sin embargo, ¿cuán grande debe ser la muestra para ser considerada representativa de las tallas del lance?

Existen pocos trabajos sobre el estudio del tamaño de muestra de frecuencias de tallas. La mayoría de ellos utilizan datos o simulaciones de desembarque en puerto (e.g. Hennemuth 1957, Schweigert \& Sibert 1983, Wild 1994, Andrew \& Chen 1997). Algunos de estos trabajos encontraron que el número de individuos no tenía un efecto importante sobre la varianza de la media de las tallas de los desembarques, pero sí el número de bodegas (e.g. Hennemuth 1957, para atún de aleta amarilla Neothunnus macropterus y barrilete Katsuwonus pelamis; Wild 1994, para simulaciones de desembarque de atún de aleta amarilla Neothunnus macropterus). Otros trabajos, como el Andrew y Chen (1997) y Montenegro Silva (2009), estudiaron la sensibilidad de las frecuencias por tallas por marca de clase (de abulón Haliotis rubra y langostino colorado Pleuroncodes monodon, respectivamente) al esfuerzo de muestreo (número de días de buceo y de viajes muestreados, respectivamente). Encontraron que, al aumentar el esfuerzo de muestreo el error en la estimación de la frecuencia relativa de las tallas disminuía. Utilizaron la convergencia en la suma de cuadrados del error como un indicador para elegir el tamaño óptimo de muestra. Ninguno de los trabajos realizados puso énfasis en el efecto del tamaño de la muestra sobre la precisión de la estimación del porcentaje de juveniles de las especies estudiadas. Sin embargo, el porcentaje de juveniles es una variable clave para el manejo adaptativo en las pesquerías (Suuronen \& Sardá 2007).

En el presente trabajo determinamos el tamaño de muestra óptimo del número de individuos a muestrear en cada lance en la pesquería de cerco de anchoveta para obtener: (1) una distribución representativa de las tallas de la captura y (2) una proporción de juveniles representativa de las capturas. Para ello nos basamos en datos biométricos registrados in situ por observadores a bordo de embarcaciones y utilizamos la suma de cuadrados del error como uno de los criterios para elegir el tamaño óptimo de muestra.

En la siguiente sección describimos los datos y la metodología empleada para el análisis. Luego, presentamos los resultados para cada lance analizado. Finalmente, damos recomendaciones prácticas para decidir sobre el tamaño de muestra en distintos escenarios.

\section{Materiales y métodos}

El programa de observadores a bordo PBP cuenta actualmente con 50 observadores. Mientras la temporada de pesca esté abierta, cada observador debe instalarse a bordo de una embarcación de la flota de cerco y permanecer en ella durante el número de días de trabajo asignado por mes ( -20 días). Durante cada viaje, el observador anota en una ficha denominada "Bitácora de Pesca" la información sobre el viaje, los lances y la embarcación.

Para este trabajo, se le pidió a 5 observadores a bordo o "bitacoreros" que cada uno muestree 1000 anchovetas en un lance. Se consideró un máximo de 1000 dado que una cantidad mayor podría representar un trabajo muy difícil para el observador. Este muestreo se realizó en abril del 2014.

La metodología propuesta de análisis se detalla a continuación.

Consideremos un observador a bordo $x$ que muestreó 1000 individuos. A esos 1000 individuos los consideramos como la población de anchovetas del lance. Luego, se sacó una muestra aleatoria $n$ de esa población. Por ejemplo, 20. Después se comparó:

La distribución de tallas de la población de 1000 individuos con la distribución de tallas de la muestra. Se determinó mediante la prueba estadística U de Mann-Whitney (Siegel 1956) si ambas distribuciones eran iguales. La hipótesis nula fue que ambas muestras (la población de 1000 y la muestra de $n$ individuos) provenían de una misma población.

La proporción de juveniles en la población de 1000 individuos con la proporción de juveniles en la muestra. Se deter- 
minó mediante una prueba binomial (Siegel 1956) si se podía considerar que ambas proporciones eran iguales.

Para eliminar el efecto de que sea precisamente esa muestra la que afectó los resultados, se hicieron varias réplicas de selección de muestra aleatoria $n(\mathrm{r}=1000$, donde $\mathrm{r}$ es el número de réplicas). Para cada réplica se realizó la comparación de distribuciones de talla y de proporción de juveniles. Luego se calculó la proporción de rechazos de la hipótesis nula para la prueba $U$ de Mann-Whitney y para la prueba binomial, con respecto al total de réplicas.

Para probar distintos tamaños de muestra, definimos $n=$ $\{25 ; 50 ; 75 ; \ldots ; 1000\}$. Es posible que para tamaños de muestra pequeños haya mucha variabilidad en la proporción de juveniles estimada. Por ello, se calculó el error cuadrático medio (ECM) de las proporciones de juveniles estimadas en las réplicas. Luego, se identificó el mínimo $n$ a partir del cual el valor del ECM es menos del 5\% del valor máximo.
Para tomar en cuenta casos reales con distribuciones de tallas distintas en los lances, pedimos a varios observadores (no sólo a uno) que muestreen 1000 individuos. En total, se realizaron 5 muestreos de más de 1000 individuos $(1008,1017,1005,1007$ y 1012, respectivamente). Para cada uno de ellos, se realizaron todos los pasos anteriores.

\section{Resultados}

Las 5 poblaciones tuvieron distribuciones de tallas distintas (Fig. 1), con 0, 0.1, 2.5, 9.1 y $31.2 \%$ de juveniles, respectivamente. Para cada uno de estos 5 casos, se presentan los resultados de la prueba U de Mann-Whitney, de la prueba binomial y del ECM (Fig. 2 y Fig. 3). Para cada una de las poblaciones y respecto de cada hipótesis (sobre distribución de tallas y proporción de juveniles), elegimos un tamaño muestral óptimo igual al mínimo tamaño de muestra que se necesita para que la proporción de rechazos de la hipótesis sea siempre menor a 0.01 (Tabla 1). En cuanto a la prueba para la distribución de tallas, la proporción

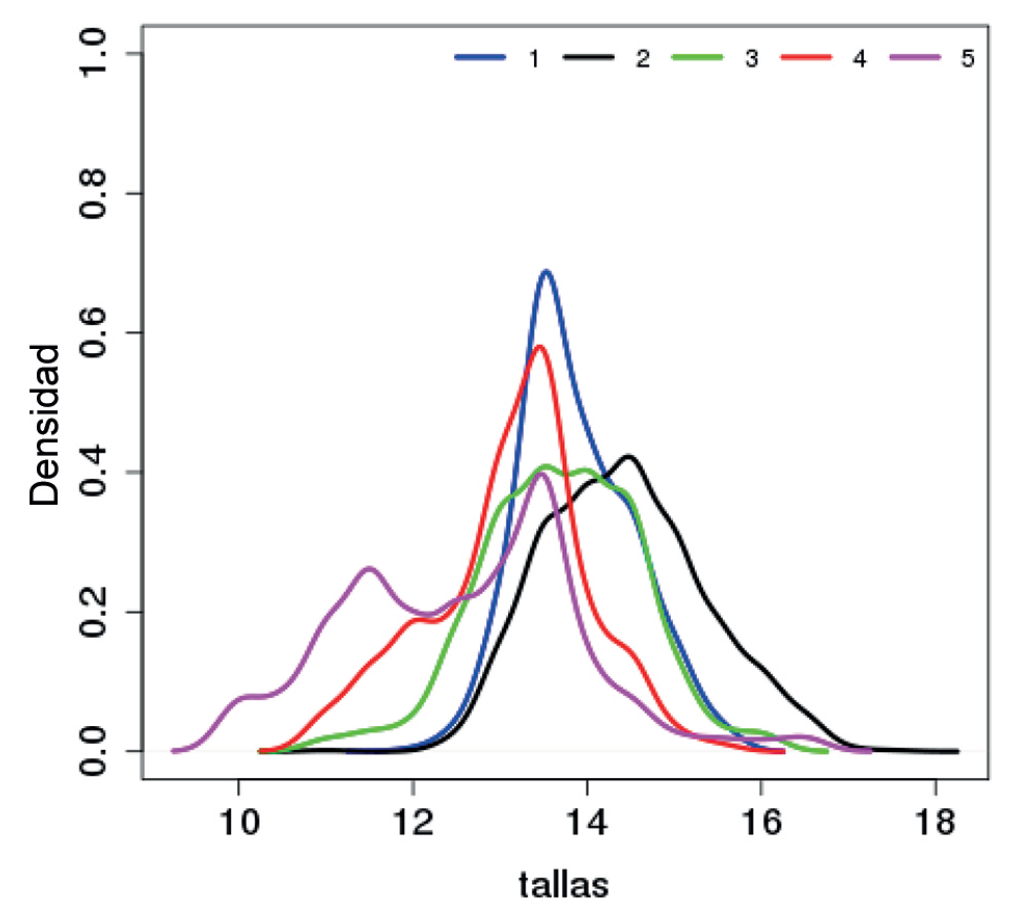

Figura 1. Distribución de tallas en las calas. Cada línea representa una población distinta; i.e. corresponde a datos tomados por observadores distintos.

Tabla 1. Resultados del tamaño de muestra óptima para la estimación de la proporción de juveniles y de la distribución de tallas para cada una de las poblaciones.

\begin{tabular}{|c|c|c|c|c|c|}
\hline & Población 1 & Población 2 & Población 3 & Población 4 & Población 5 \\
\hline Porcentaje de Juveniles & $0.0 \%$ & $0.1 \%$ & $2.5 \%$ & $9.1 \%$ & $31.2 \%$ \\
\hline $\begin{array}{l}\text { Tamaño óptimo de muestra para la } \\
\text { distribución de tallas }\end{array}$ & 350 & 300 & 300 & 300 & 300 \\
\hline $\begin{array}{l}\text { Tamaño óptimo de muestra para la } \\
\text { estimación de proporción de juveniles }\end{array}$ & -- & 75 & 350 & 400 & 500 \\
\hline $\begin{array}{l}\text { Tamaño óptimo de muestra para la } \\
\text { precisión en proporción de juveniles }\end{array}$ & -- & 125 & 125 & 125 & 100 \\
\hline
\end{tabular}


(a)

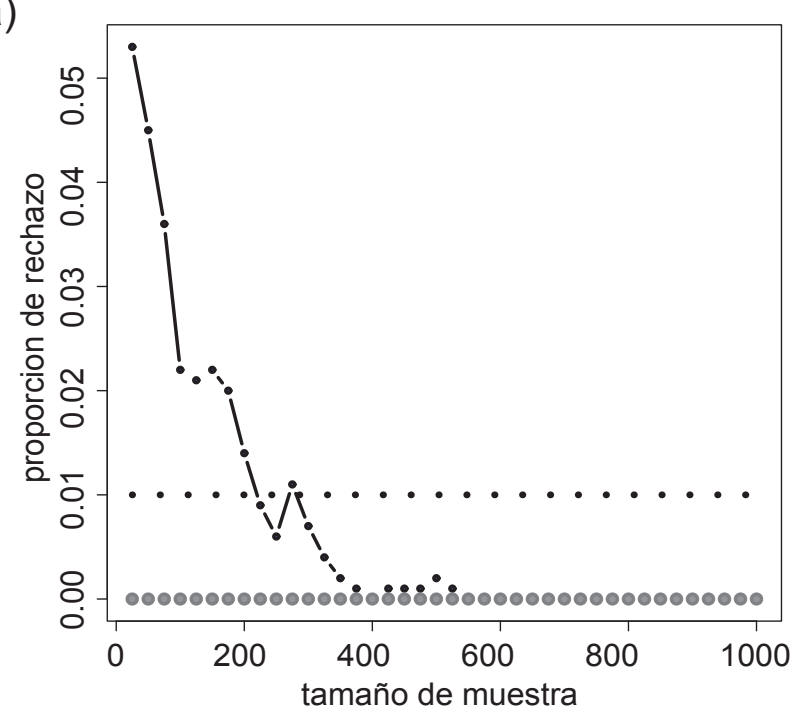

(c)

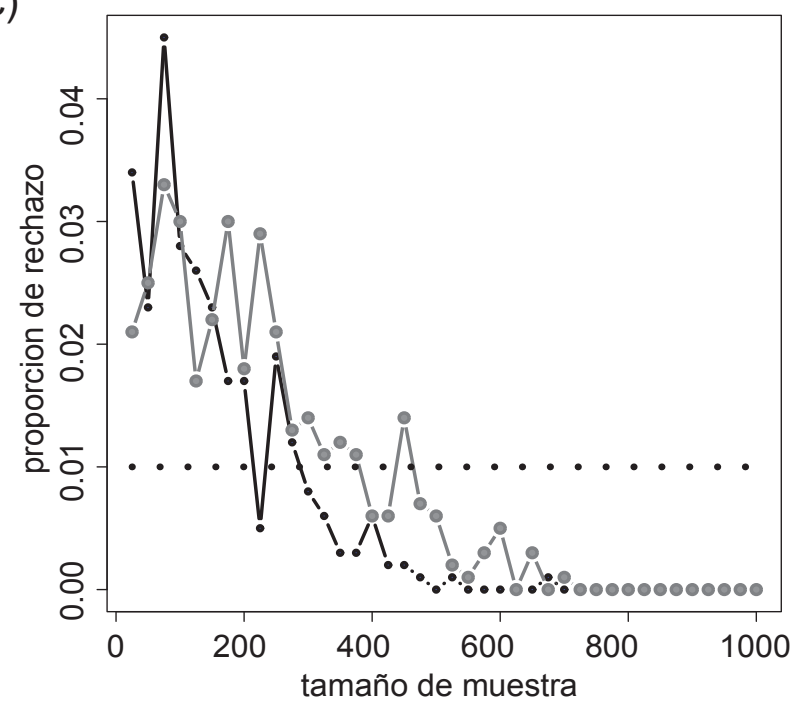

(b)

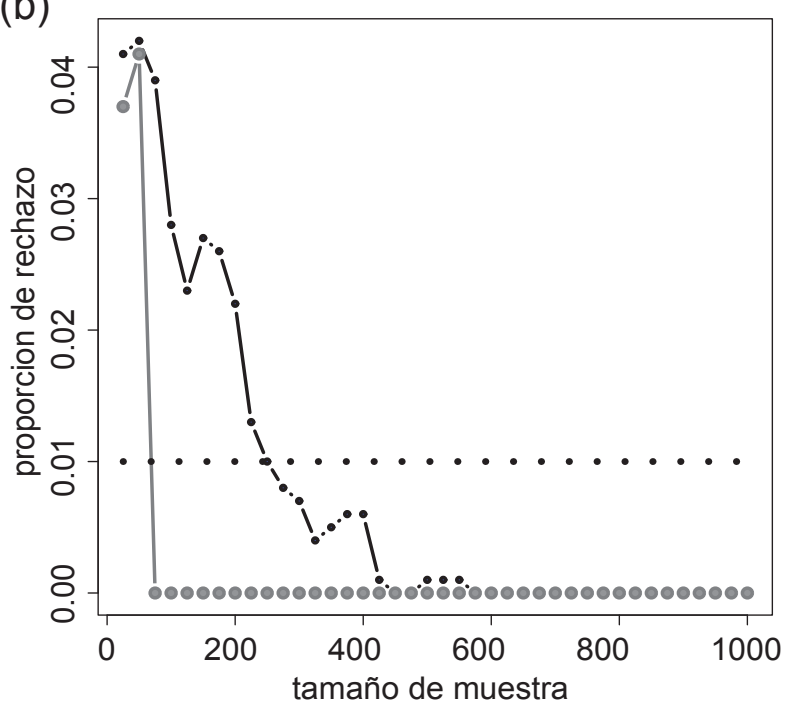

(d)

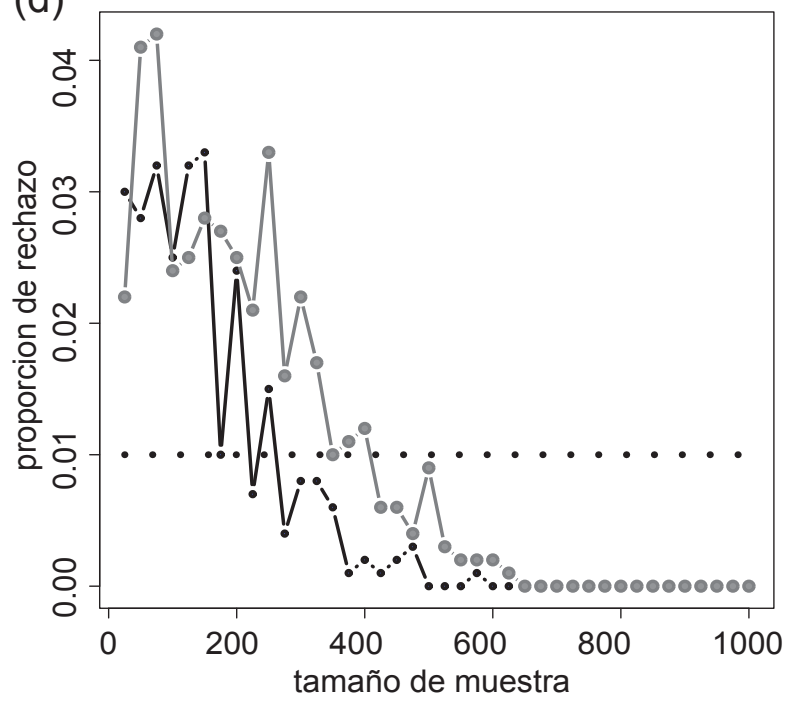

(e)

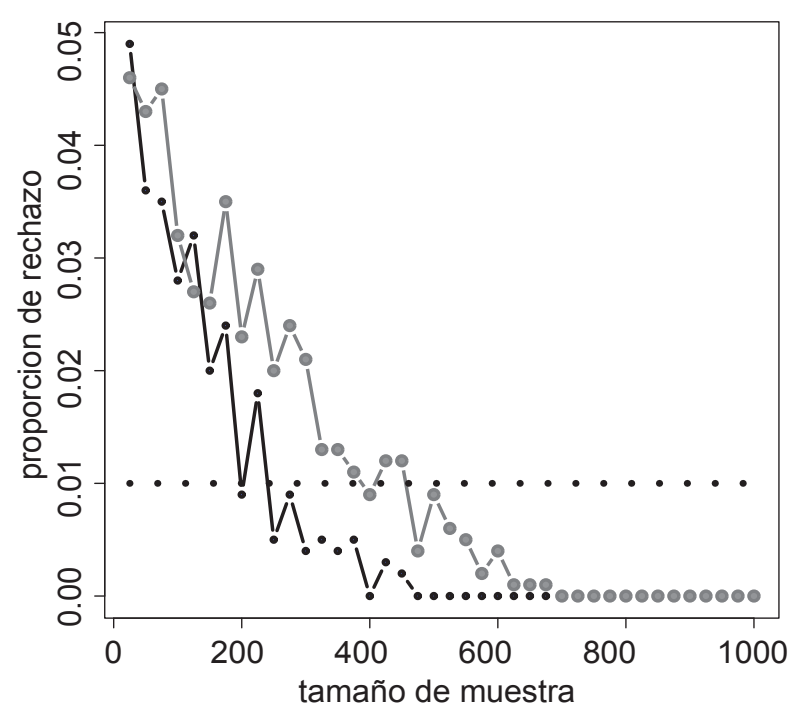

Figura 2. Resultados de las pruebas de comparación de distribuciones de tallas y proporciones de juveniles. Para cada población ( $a, b, c, d$ y e), cada punto de la línea azul corresponde a la proporción de réplicas en las que se rechazó la hipótesis nula de distribución de tallas iguales, para cada tamaño de muestra. A su vez, cada punto de la línea roja corresponde a la proporción de réplicas en las que se rechazó la hipótesis nula de igual en la proporción de juveniles de la muestra respecto a la población. La línea horizontal negra punteada corresponde al límite de proporción de rechazos que se dispone aceptar, igual a 0.01. 
a)

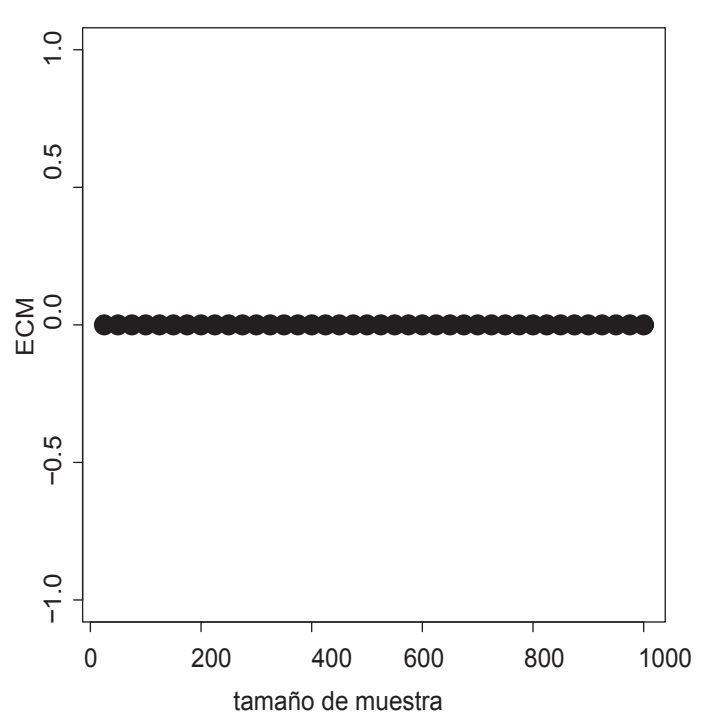

c)

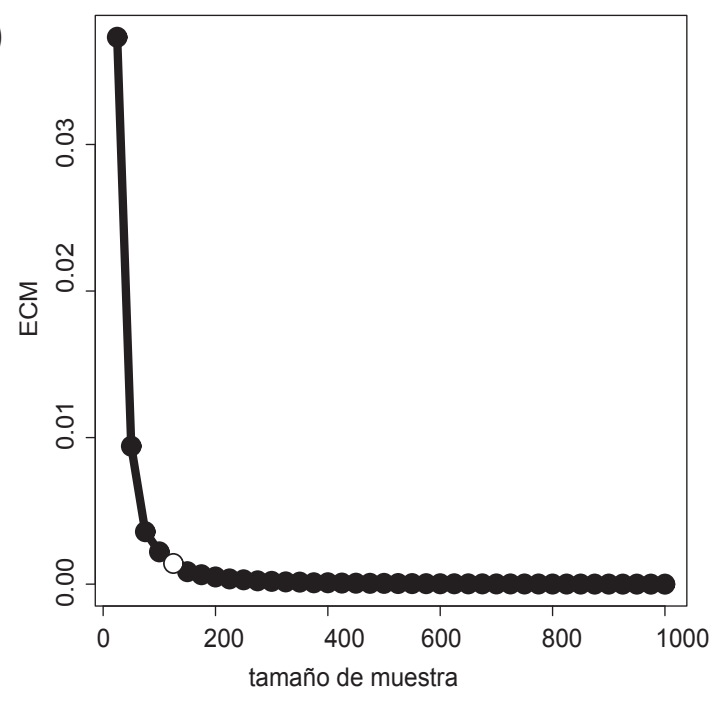

b)

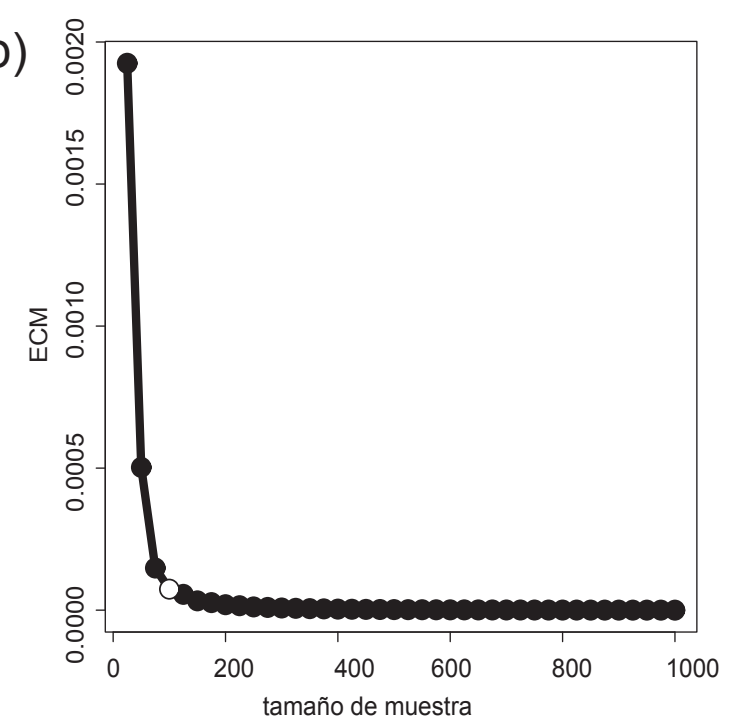

d)

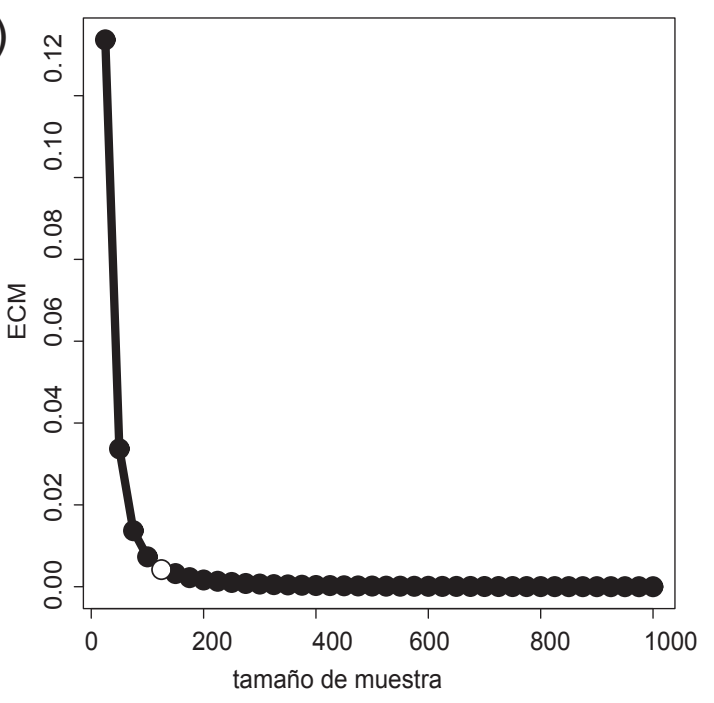

e)

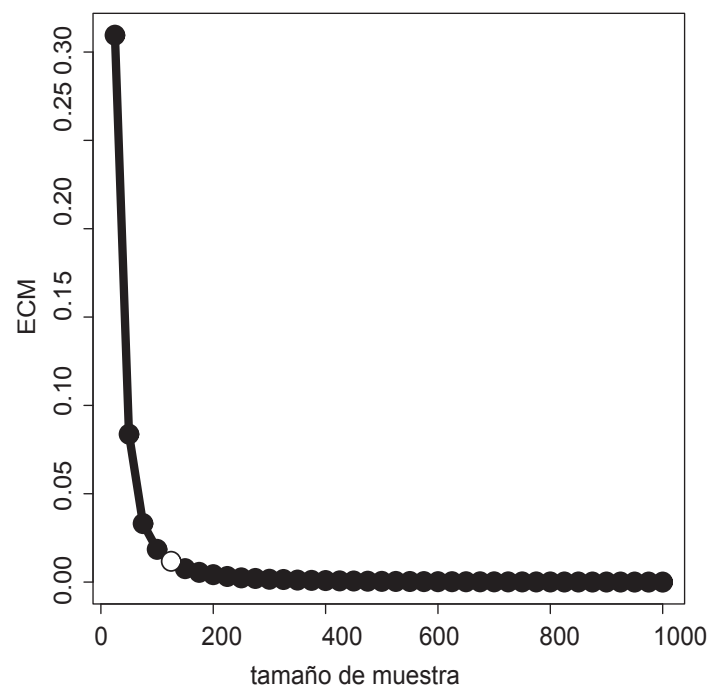

Figura 3. Valores de error cuadrático medio (ECM) para las proporciones de juveniles para cada tamaño de muestra. Para cada población ( $a, b, c, d$ y e), el punto azul corresponde a aquel tamaño de muestra a partir del cual el valor del ECM es menos del $5 \%$ del ECM máximo. 
de rechazo para la prueba para cada tamaño de muestra fue similar para todas las poblaciones (Fig. 1). Es decir, el rechazo de la prueba fue sensible al cambio en el tamaño de muestra, más que a la distribución de tallas de la población. Por el contrario, la hipótesis de igualdad de proporciones de juveniles entre la muestra y la población sí requirió mayor tamaño de muestra para ser aceptada para poblaciones con más juveniles. Con el criterio de ECM se observó que al menos se necesitan muestrear 125 individuos para obtener un valor pequeño de ECM (i.e. convergencia en el valor de ECM) en 3 de los casos, y 100 en uno de ellos (Tabla 1).

Para la población 1 no hubo un tamaño óptimo de muestra para estimar la proporción de juveniles puesto que en esa población (i.e. ese lance) no hubo juveniles. El tamaño varía entre 75 individuos para la población 2 de $0.1 \%$ de juveniles y 500 individuos para la población 5 de $31.2 \%$ de juveniles.

\section{Discusión}

En este trabajo, estimamos el número óptimo de anchovetas a muestrear en cada lance para lograr dos objetivos: una distribución representativa de la distribución de tallas del lance, y una proporción de juveniles que sea representativa del lance.

En trabajos realizados para otras pesquerías, Hennemuth (1957) y Wild (1994) encontraron que el número de individuos no tuvo un fuerte efecto en la estimación de la media de las tallas en sus análisis. Nuestros resultados son coherentes con estos trabajos en el sentido de que la proporción de rechazos de la prueba U de Mann-Whitney fue menor a 0.1 en todos los casos (todos los lances y todos los tamaños de muestra). Sin embargo, dado que las distribuciones de tallas se utilizan para el manejo de la pesca, se fijó un umbral de rechazo de 0.01 , lo que significa que el riesgo de obtener una frecuencia de tallas distinta a la de la totalidad del lance es de menos de una vez cada 100. Se necesitó muestras de al menos 300 para quedar debajo de ese umbral.

Como el estadístico U de Mann-Whitney es calculado a partir de rangos (Siegel 1956), toda la distribución de las tallas es tomada en cuenta para el análisis. Por ello, los resultados aquí descritos pueden considerarse más robustos que los trabajos sobre estimación de la media y varianza de las tallas. Asimismo, este trabajo presenta un valor agregado a lo propuesto por Andrew y Chen (1997) al aplicar pruebas estadísticas para determinar si las diferencias respecto a las poblaciones son significativas, además de utilizar la suma de cuadrados del error.

Mientras la calidad de la representatividad de la distribución de tallas no se vio afectada por la cantidad de juveniles en el lance, la estimación del porcentaje de juveniles sí. Eso quiere decir que si hay un porcentaje alto de juveniles y tomamos una muestra pequeña, podríamos no obtener un número representativo de juveniles en la muestra y equivocarnos en la estimación.

Como recomendación práctica, se plantea que en general se muestree un mínimo de 300 individuos por cala, ya que en casi todos los lances analizados se necesitaron muestrear 300 individuos para estimar correctamente la distribución de tallas. Por otro lado, en escenarios de alta proporción de juveniles, esperados a partir de informes de cruceros o detectados a partir de datos recientes de observadores a bordo, se aconseja incrementar el tamaño de muestra a 500. También se recomienda que en ningún escenario se muestree menos de 125 individuos, para asegurar un nivel mínimo de precisión en la estimación de la proporción de juveniles.

En los manuales de recolección de datos (Bouchon et al. $1998,2001)$ se indica que la muestra debe componerse de 120 a 160 individuos, aunque en la práctica la mediana del tamaño de muestra es 178. El incremento que se propone en este trabajo para el número de individuos muestreados representa un costo en tiempo y esfuerzo para el observador, pero partimos del supuesto que este costo no disminuye la calidad de su trabajo ni lo dificulta de manera significativa. En futuros trabajos se debe analizar la relación costo-beneficio del incremento en tamaño de muestra (e.g. costo en tiempo versus beneficio en significancia estadística).

Este trabajo se ha realizado bajo el supuesto de que las muestras que se toman son aleatorias. Es decir, que el balde de $2 \mathrm{~kg}$ de muestra que toma el observador no representa ningún sesgo en cuanto a la distribución de tallas del lance. No existe evidencia que haga suponer lo contrario. Además, análisis estadísticos realizados previamente indican que tomar la muestra al inicio, mitad o fin del lance no produce una diferencia sistemática en la distribución de tallas encontrada.

Una alternativa al muestreo aleatorio podría ser el muestreo por cuotas (Moser 1952). Es un muestreo no probabilístico que consiste en definir estratos (i.e. intervalos de tallas) y fijar un número de individuos a muestrear para cada estrato. Este número suele ser proporcional a la cantidad de individuos que pertenecen a ese estrato en la población. Sin embargo, las características demográficas de los stocks pueden variar dependiendo del éxito en la reproducción, de la mortalidad natural y por pesca. Por lo tanto, se necesitarían métodos apropiados para tomar en cuenta estos efectos y así actualizar la estimación de la cuota por estrato de manera frecuente. Al ser el ecosistema de Humboldt altamente variable a múltiples escalas espaciales y temporales (Bertrand et al. 2004, 2011, Chavez et al. 2008, Arias-Schreiber et al. 2011), realizar estos cálculos con exactitud es aún más complicado.

El objetivo del presente trabajo fue determinar un tamaño de muestra de individuos para la distribución de tallas y proporción de juveniles en un lance. Es importante resaltar que la metodología aplicada en este trabajo puede utilizarse para muestreos biométricos de distintas especies, para observaciones a bordo o en tierra.

Una pregunta que escapa del alcance de este trabajo, pero que valdría la pena responder en trabajos futuros sería cómo realizar un diseño de muestreo que garantice que los lances muestreados sean representativos de manera que las distribuciones de tallas obtenidas nos brinden información de la distribución de tallas del stock. Existen diversos trabajos que utilizan muestreos multietápicos para ese fin (e.g. Hennemuth 1957, Montenegro Silva 2009), pero antes de realizar un trabajo teórico al respecto se debe revisar si es factible poner en práctica un diseño de esa naturaleza en la pesquería pelágica de cerco peruana.

\section{Agradecimientos}

Agradecemos a Julio Limanche por la coordinación para la toma de datos y a los bitacoreros, sin cuya labor, todo esto no sería posible. También damos gracias a Gersson Román y Paul Tacuri por la ayuda con la documentación sobre el procedimiento de muestreo en el Programa Bitácoras de Pesca. 


\section{Literatura citada}

Andrew N.L. \& Y. Chen. 1997. Optimal sampling for estimating the size structure and mean size of abalone caught in a New South Wales fishery. Fishery Bulletin 95:403-413.

Arias Schreiber M., M. Niquen \& M. Bouchon. 2011. Coping strategies to deal with environmental variability and extreme climate events in the Peruvian anchovy fishery. Sustainability 3:823-846. https://doi.org/10.3390/su3060823

Bertrand A., A. Chaigneau, S. Peraltilla, J. Ledesma, M. Graco, F. Monetti \& F. Chavez. 2011. Oxygen: a fundamental property regulating pelagic ecosystem structure in the coastal southeastern tropical Pacific. PLOS ONE 6:e29558. https:// doi.org/10.1371/journal.pone.0029558

Bertrand A., M. Segura, M. Gutiérrez \& L. Vásquez. 2004. From small-scale habitat loopholes to decadal cycles: a habitatbased hypothesis explaining fluctuation in pelagic fish populations off Peru. Fish and Fisheries 5:296-316. https:// doi.org/10.1111/j.1467-2679.2004.00165.x

Bouchon M., M. Niquen, M. Arias-Schreiber \& R. Bello. 1998. Manual de operaciones del proyecto bitácoras de pesca. Informe Progresivo 74. Instituto del Mar del Perú.

Bouchon M., M. Niquen, J. Mori, A. Echevarría \& S. Cahuín. 2001. Manual de muestreo de la pesquería pelágica. Informe progresivo 157. Instituto del Mar del Perú.

Chavez F., A. Bertrand, R. Guevara-Carrasco, P. Soler \& J. Csirke. 2008. The northern Humboldt Current System: Brief story, present status and a view towards the future. Progress in Oceanography 79:95-105. https://doi.org/10.1016/j. pocean.2008.10.012

Di Natale A., J. De La Serna, G. De Metrio, V. Restrepo, A. Srour \& G. Tserpes. 2012. On the reduction of juvenile swordfish catches in the Mediterranean. Col.Vol.Sci.Pap. ICCAT, 54:1529-1533.

Díaz E. 2013. Estimación del crecimiento del jurel Trachurus murphyi capturado en el Perú, a partir del análisis de las frecuencias por tallas. En: Csirke J., R. Guevara-Carrasco \& M. Espino (Eds.). Ecología, pesquería y conservación del jurel (Trachurus murphyi) en el Perú. Revista Peruana de Biología número especial 20(1):061-066 https://doi.org/10.15381/ rpb.v20i1.2620

Hennemuth R. 1957. An analysis of methods of sampling to determine the size composition of commercial landings of yellowfin tuna (Neothunnus macropterus) and skipjack (Katsuwonus pelamis). Inter-american tropical tuna commision, Bulletin 2:174-225.
Montenegro Silva C. 2009. Tama-os de muestra para estimar la estructura de tallas de las capturas de langostino colorado en la zona centro-norte de Chile: una aproximación a través de remuestreo. Latin American Journal of Aquatic Research 37:161-180. https://doi.org/10.3856/vol37issue2-fulltext-4

Moser C. A. 1952. Quota sampling. Journal of the Royal Statistical Society. Series A 115:411-423. https://doi. org/10.2307/2980740

Oliveros-Ramos R. \& E. Díaz. 2015. Estimación de la captura total permisible del stock norte-centro de la anchoveta peruana. Protocolo IMP/DGIRP-AFDPERP. Edición 2 revisión 3, abril 2015.

Palomares M., P. Muck, J. Mendo, E. Chumán, O. Gómez \& D. Pauly. 1987. Growth of the Peruvian anchoveta (Engraulis ringens), 1953 to 1982. In D. Pauly and I. Tsukayama (eds.). The Peruvian anchoveta and its upwelling ecosystem: three decades of change. ICLARM studies and reviews 15, $351 \mathrm{p}$.

Pauly D. \& G. Morgan (editors), 1987. Length-based methods in fisheries research. ICLARM Conference Proceedings 13, 468 p. International Center for Living Aquatic Resources Management, manila, Philippines, and Kuwait Institute for Scientific Research, Safat, Kuwait.

PRODUCE. 2014. Boletín Estadístico Pesquero 2014. Dirección de Estudios y Derechos Económicos Pesquero y Acuícolas. Ministerio de la Producción, 65p.

Quinn T. \& R. Deriso. 1999. Quantitative fish dynamics. Oxford University Press, New York, N.Y.

$\mathrm{RM} \mathrm{N}^{\circ}$ 215-2015-PRODUCE. Resolución que suspende las actividades extractivas del recurso anchoveta y anchoveta blanca entre los $13^{\circ} 30^{\prime}$ y $13 \quad 59^{\prime} \mathrm{S}$ y desde las 5 a las 20 mn, a partir del 28 de junio 2015 y por 05 días calendarios.

Schweigert J.F. \& J.R. Sibert. 1983. Optimizing survey design for determining age structure of fish stocks: an example from British Columbia Pacific Herring (Clupea harengus pallasi). Canadian Journal of Fisheries and Aquatic Sciences 40:588597. https://doi.org/10.1139/f83-078

Siegel S., 1956. Nonparametric statistics for the behavioral sciences. McGraw-Hill, New York.

Suuronen P. \& F. Sardá. 2007. The role of technical measures in European fisheries management and how to make them work better. ICES Journal of Marine Science, 64: 751-756. https://doi.org/10.1093/icesjms/fsm0 49

Wild A. 1994. An evaluation of length-frequency sampling procedures and subsequent data analysis for purse seine-caught yellowfin tuna in the Eastern Pacific Ocean. Inter-american tropical tuna commision, Bulletin 21:3-43. 
